\title{
Understanding Parents' Mistakes in Insulin Doses for Hyperglycaemia Treatment
}

\author{
Wala'a Barifah ${ }^{1,2}$, Bill Davey ${ }^{2}$, Vince Bruno ${ }^{2}$ \\ ${ }^{1}$ College of Admins and Financial Sciences, Taif University, Taif, Saudi Arabia \\ ${ }^{2}$ School of Business IT \& Logistics, RMIT University, Melbourne, Australia
}

Email address:

Walaa.Barifah@rmit.edu.au (W. Barifah),Bill.Davey@rmit.edu.au (B. Davey), Vince.Bruno@rmit.edu.au (V. Bruno)

To cite this article:

Wala'a Barifah, Bill Davey, Vince Bruno. Understanding Parents' Mistakes in Insulin Doses for Hyperglycaemia Treatment. Science Journal of Public Health. Vol. 6, No. 3, 2018, pp. 91-99. doi: 10.11648/j.sjph.20180603.15

Received: April 4, 2018; Accepted: May 18, 2018; Published: June 21, 2018

\begin{abstract}
This study focuses on how parents make mistakes when administering insulin doses in treating hyperglycemia in their T1D diabetic children. This research employed a qualitative approach, conducting 58 semi-structured, in-depth interviews with 37 Saudi Arabian and Australian parents with T1D children, and 20 combined health professionals and company representatives. A conceptual tree analysis of the interviews was carried out using Atlas software. The initial coding was analyzed into two major themes: Misestimations and misunderstandings of insulin correction doses; and Mistakes in using injection tools and techniques. Each of these themes affords insight into correcting parents' mistakes in insulin doses through a variety of techniques and technologies for T1D-related insulin delivery. In general, parents had made both corrected and uncorrected mistakes, and surprisingly, many were unfamiliar with the focal meaning of correction doses and how they are estimated or used. Despite significant efforts in diabetes education, the incidence of adverse health outcomes continues. This study shows that parental mistakes in insulin doses for treating hyperglycemia are crucial and involved issues. There are many ways to address these mistakes, and this study seeks to provide recommendations in order improve diabetes care practices.
\end{abstract}

Keywords: Insulin Dosage, Parent, Type 1 Diabetes, Mistake, Hyperglycemia

\section{Introduction}

Diabetes is an increasingly prevalent disorder caused by poor production of insulin in the body. When diabetes causes the body's glucose level to rise, this leads to a condition known as hyperglycemia. Diabetes mellitus occurs in two different forms that include type one (insulin dependent) and type two (non-insulin dependent) [1]. Type one diabetes (T1D) is a chronic autoimmune disease that affects the pancreas by destroying beta cells within the islets of Langerhans. The pancreas is responsible for synthesis and secretion of insulin which controls glucose levels [2]. After beta cells are destroyed, the blood sugar level is no longer controlled and patients require insulin replacement in the body. Daneman [3] notes that the primary goal of treating diabetes by insulin is keeping the blood glucose (BG) levels close to target levels, $6 \bullet 0-12 \bullet 0(\mathrm{mmol} / \mathrm{L})$. Unfortunately, inaccurate insulin doses, immoderate, misestimated and overlapping doses puts T1D patients particularly children - at risk when treating hyperglycemia.

According to Roemer [4], there is no single drug that can treat every aspect of diabetes mellitus due to the complexity of beta cells in the pancreas. The medication available is used with a combination of lifestyle to maintain blood sugar levels and reduce hyperglycemia. Wolfsdorf et al [5] note that untreated hyperglycemia sometimes progresses into diabetic ketoacidosis (DKA) which is a serious complication. In the case of DKA, the body runs out of insulin that supports glucose entering the cells. Kanikarla-Marie and Jain [6] note that the body switches to bringing fatty acids and bludge, producing toxic acidic, ketone. Subsequently, an intervention to balance the $\mathrm{BG}$ levels by taking extra insulin doses is necessary. According to Hanas [7], the treatment process is selected according to the pathophysiology of the individual.

Despite people's efforts to control diabetes, sometimes glucose levels are hard to control. A small amount of insulin is required by the body in order to control blood glucose levels in the body. An individual whose pancreas does not produce enough insulin to control blood sugar will need 
treatment in order to control blood sugar levels [8]. People should have basic knowledge of insulin usage in the body. For instance, about $50 \%$ of the total daily insulin dose replaces overnight insulin. Caregivers need to provide an appropriate insulin dose regimen to the patients. The regimen provides formulas that allow patients to calculate how much to adjust insulin doses taken at meals or when correcting high blood sugars. Hulkower et al [9] note that some insulin dosing requirements are confusing for parents who are responsible for dosing their children.

Diabetes therapy is complicated and managing hyperglycemia with the correct insulin doses is challenging. Errors of insulin administration occur with parents, patients and even medical professionals [10]. When the insulin correction is administered wrongly, this unfortunately can causes injury or harm [11]. According to the ADA, in 2011, approximately 175,000 people were admitted to an emergency department because of a hyperglycemic crisis.

Trief et al [12] note in their study on insulin correction doses that participants reported errors commonly occurring when Blood Glucose (BG) required a correction dose. However, only $6.7 \%$ of the participants knew about and were using the algorithm of insulin-to-carbohydrate (I:C) ratios to determine the required insulin doses for their bodies. By contrast, $13.3 \%$ of participants "never" used the I:C ratios for insulin correction doses.

Trief et al's study [12] further notes that nearly half of the participants, $48.3 \%$, reported they never balanced the insulin doses to correlate with the BG reading. The study found that the level of diabetes awareness related-numerical especially in insulin doses, was low and that could be a factor helping to facilitate the occurrence of injected errors. Thus investigating mistakes in insulin doses that cause hyperglycemia to reduce errors has been ignored by the research community. This paper focuses on mistakes of parents who are not confident in administering the correct dosage for treating hyperglycemia. Also examined through interviews, are their reactions to managing hyperactivity once they treat the condition.

\section{Literature Review}

The review of past and related literature aims to answer the research problem by identifying objectives and establishing the study's questions. The researcher will identify different relevant informational materials from primary and secondary sources in relates to realize insulin therapy and parents' mistakes concerning insulin doses for T1D children. Academic materials and other publications on the study field will be used to fill the research gap by concentrating on parents' mistakes regarding insulin dosages, hyperglycemia treatment on T1D children, and any subsequent chronic diseases. The literature on case studies and background information on hyperglycemia and its treatment makes it possible to examine the issues. Most parents find it hard to recognize insulin dosages of their diabetics children when treating high blood sugar.

\subsection{Parents' Mistakes Concerning Insulin Dosages}

Umpierrez et al [13] note that modifying to the insulin dosage regimen is a skill which takes time health providers and patients to master. Understanding the insulin dosage is essential to parents who have a charge of caring for their T1D children. When blood sugar level is high or low, it commonly becomes more difficult to understand which type of insulin, bolus or basal to readjust and by how much. Diagnosis of T1D in children influences not only the diabetic but also the family [14]. Parents have a tremendous responsibility to ensure the blood sugar (BS) level of the children is controlled. Parents have to cope with various situations when treating high BS such as self-doubt, less family activities, apprehension and declining quality of life.

Preiser [15] observes that most people believe that diabetes is a chronic illness that only affects adults. However, this is not the case since T1D stays an infectious disorder in children and adolescents while T2D affects adults. Parents still make mistakes about children's diabetes and how therapy processes should be conducted. Parents should be able to identify different symptoms and signs in children who cannot express what they are feeling [16]. Although the T1D develops gradually, signs manifest quickly. When children indicate continual signs and symptoms of vomiting, nausea, weight loss and abdominal pain, parents must take them to the hospital for diabetes diagnosis. Pettitt et al [17] attention to that immediate treatment helps saves children's lives since an sudden death can occur very quickly, even in less than one week or month.

Parents must be aware of seeking medical assistance for children with T1D. Niedel et al [18] remark that patients with T1D need lifelong insulin therapy. Most patients require a few injections of insulin on a daily basis. The doses should be adjusted in order to control the glucose level in a patient's blood. Most diabetics are entitled to insulin injection for life which becomes a difficult situation for parents. According to Young-Hyman and Peyrot [19], at some point parents are afraid of injecting insulin into their children because too much insulin can put the child in a coma or result in death. Further, parents have such huge responsibilities that are sometimes very difficult - if not impossible - to achieve. Wolfsdorf et al [5] state that despite administering insulin dosage to the diabetics, parents have to manage diet, measure blood sugar level and ensure they engage in physical exercises.

\subsection{Parents and Hyperglycaemia Treatment and Mistakes}

Turchin, Matheny, and Shubina [20] note that hyperglycemia treatment in children starts with diagnosis at an early age. A random blood sugar test is done in order to detect if the child is diabetic. Regardless of when the child ate, random blood sugar level of 200 milligrams per deciliter $(\mathrm{mg} / \mathrm{dL})$ or higher suggests diabetes. Glycated hemoglobin (A1C) test reflects the child's average blood sugar for the past three months. The A1C test determines the percentage of BS attached to hemoglobin in red blood cells. A glycated 
hemoglobin level of $6.5 \%$ or higher on separate tests to signify diabetes [21]. As well, a fasting BS test is necessary to detect if the child has diabetes. A sample of blood is collected after a child fasts overnight whereby the level of $126 \mathrm{mg} / \mathrm{dL}$ or higher assure diabetes.

After diagnosis children need regular follow-up appointments to ensure proper management of diabetes. Doctors periodically use urine and blood tests to check children's kidney, cholesterol levels and thyroid function [22]. For example, the ADA recommends an A1C of 7.5 or lower for all children. Treatment of T1D in children is lifelong and has diverse management processes, which include insulin therapy and monitoring a balanced healthy diet [23]. However, these activities can sometimes overwhelm parents. For instance, parents at times manage BS level of the children well and yet at other times the process fails. These failures are serious mistakes and may only encourage parents to give up caring for their children. Umpierrez [13] states that the parents should communicate and work closely with the child's diabetes team in order to keep the patient's BS level at the recommended level.

T1D is a serious disorder and demands the parents' commitment to ensure their diabetic child receives proper medication and protection [24]. Lifestyle changes are significant and parents should always ensure that children engage in physical exercises in order to decrease BS levels. Parents' efforts are worthwhile particularly if they do not misunderstand insulin dosage and are not scared of the diabetes therapy process. Parents have the responsibility to encourage grown-up children to take an active role in their own diabetes management [25]. Melissa Spezia in [26] recommended different approaches to rural Midwest families in the United States on how to manage diabetic children. The techniques comprised helping children regarding good food choices, physical activeness, knowing how to test blood sugar and inject insulin.

\subsection{Mistakes of Parents Regarding Chronic Diseases}

According to Bruno et al [27], living with a patient suffering from a chronic disease can result in challenges that are above the usual parenting responsibilities because special demands that result in social and financial constraints. Parents raising children with many chronic diseases have to cope with difficult situations and ensure they survive [28]. They need to alter expectations and understand more about the complications affecting the child. Sometimes the mothers and fathers find management of the chronic disease difficult and succumb to depression and stress. . Correcting a mistake is a good initiative that will assist parents who are determined to offer care services to their T1D children [29]. It means they are diligent in trying to save the life of the patient from a chronic disease.

T1D is a chronic disease diagnosed in young and usually has severe impacts on the family. Parents exhibit various behaviours when handling their children [30]. The most reported mistakes parents make regarding T1D children include concerns about complications, grief and fear. Parents are entirely responsible for managing children's diabetes and this puts much pressure on them. Diabetes mellitus (DM) is a common childhood disease that affects a large number of children in the United States [31] and especially much more recently [32] \& [33]. T1D is associated with both short- and long-term complications. For instance, hypoglycemia (low BG level) is a fatal short-term complication that results from poor physical exercise, too much insulin in the body and insufficient carbohydrate intake. According to Bruttomeso and Grassi [34], the complication is manifested by confusion, brain damage, weakness or even death. Long-term problems include renal, immune suppression and cardiovascular diseases.

Faulkner [26] describes family mistakes concerning school-age diabetics and parents' influence as well as those children implementing self-care. Interviews were conducted with seven families living in the Midwest and they documented different parents' mistakes. These mistakes demonstrated parents' misunderstanding of the insulin dosage regimen. Most parents were stressed by the scheduled daily routines and managing T1D while others were apprehensive about the insulin reactions [35]. If adolescents are diagnosed with diabetes, it is the responsibility of the fathers and mothers to take care of the condition. Parents experience emotional issues when they realize their children require insulin and blood sugar level monitoring on a daily basis. They find that it is difficult to inject insulin to their own children, accepting and explain the condition to them.

\subsection{Exploring the Mistakes of Parents in Hyperglycemia Treatment - Research Gap}

According to Dungan et al [36], T1D is common in young people in the United States below the age of twenty years. Since its diagnosis takes place at an early age, the burden of managing the complication falls on both the patient and parents. Parental involvement is crucial for proper diabetes management of the child [37]. However, because of complex activities involved in managing the condition in children, parents sometimes make mistakes in insulin dosage to their children. The underlying issues on injection dosage and how it is done is a challenging problem to parents. Most of the time parents need guidance on the amount of insulin dosage and the techniques of injecting the children. Cryer [22] highlights that parents always have many quires on the injection area and impacts on the T1D children.

During early childhood, both fathers and mothers assume responsibilities of controlling T1D in children but as they get older, they surrender these duties to the children [38]. However, in the process, some parents demonstrate mistakes in managing the condition in children. According to Marzelli et al [39], the life of a child suffering from diabetes changes abruptly, meaning that the condition requires an immediate response from the parents.

Melissa Spezia's study in [26] demonstrates that some participants blamed themselves due to a child's disease. An injected overdose of insulin into a T1D child leads to hypoglycemia which only confuses the parents more, so they 
become scare about injecting their child. Faulkner [26] also notes that at times parents feel helpless about the disorder and are unable to provide the necessary care or seek services from the medical team. The fathers and mothers at times surrender and fail to control their child's diet. However, this is not acceptable and the fathers and mothers need to encourage the patient and collaborate with the local medical team to effectively control the complication.

\section{Method}

\subsection{Sample Population}

The research question for this study is as follows: How do parents respond to misunderstanding of the way to deal with incorrect dosage for hyperglycemia treatment? A qualitative approach was adopted, because obtaining productive insights into parents' mistakes requires a deep understanding of the relevant phenomena [40]. Semi-structured questions allowed a wide range of issues to be introduced and also permitted interviews to be guided by the parents. 36 parents of Type 1 diabetic children and adolescents were included in the study as the parents are the primary care providers. One mother of a non-diabetic child was also included in the study to understand the public's awareness. The participants were drawn from two countries, Australia and the Kingdom of Saudi Arabia, to broaden the range of types of parents included.

A total of 58 interviews were conducted, and pilot interviews were held on 8 participants from the two countries. Several types of professionals were interviewed with a view to documenting their understanding of the problems. This helped to establish the mistakes parents make and provide triangulation to the study. The professionals included were pediatric endocrinologists, diabetes educators, dieticians and companies' representatives of diabetes devices. The interviews used prompting techniques as proposed by Browne and Rogich [41]. Prompting techniques provide a context to test the relative effectiveness of new techniques, and it involves asking 'Who,' 'What,' 'When,' 'Where,' 'How' and 'Why' questions. The questioning strategy adopted entails asking questions based on a theoretical model of knowledge structures. Use of prompting techniques in interviews elicits more information from participants on a given topic.

\subsection{Analysis Process}

The interview transcripts obtained were uploaded to Microsoft Word and then the Atlas Software for their management, coding, visualization, retrieval of queries and reporting data. The coding and themes obtained from the Atlas software were reviewed by two external researchers for validity checks. Participant codes started with 'para' which indicates parents, 'dia' indicates diabetes educators, 'endo' refers to endocrinologists and lastly, 'rep' indicates company representatives. The transcripts were analyzed starting with the assumption that parents' mistakes when using the insulin dosages in treating hyperglycemia.

\section{Results}

Overall, much of the data collected indicated that parents made many mistakes concerning insulin disease when treating hyperglycemia. Surprisingly, many were unfamiliar with the focal meaning of correction doses and how they are used. Two main themes were identified which described parents' mistakes with inappropriate insulin dosages, and each theme comprised a number of categories.

Theme 1: Mistakes parents make with estimating insulin correction doses

This theme provides insights into parents' difficulties in how to calculate and estimate correct doses. This theme consists of two categories: (1) underestimations or overdoses of insulin units and misusing Glucagon injection; and (2) misapplications of correction factors

Parents underestimated and overdosed insulin units and misused Glucagon injection

This mistake results from parents' dependence on their ability to estimate insulin doses without prior knowledge. Examples of two opposite scenarios appeared in the data. First, some parents gave inadequate total units of insulin because of their concern about short-term complications-for instance, hypoglycemia, which can develop into seizure and coma resulting from low blood sugar. Their concern led to their injecting their children with too little insulin, resulting in a rise in blood glucose levels. Parents' remarks included the following:

[Obviously, we want to avoid hypoglycemia at all costs, and we are not giving him too much insulin]. (Par-a-2)

[I usually lower the amount of her insulin by not giving full doses because hypo really scares me!] (Par-k-1)

On the other hand, some parents injected their children with an overdose of units because they were overwhelmed by high $\mathrm{BG}$ readings.

[High surges worry me; I gave him 10 units of insulin instead of 6 units. Then I saw him unconscious!] [Coma due to low level of Blood glucose] (Par-k-11)

This unconsciousness as stated in the quote immediately above was the result of hypoglycemia. In addition, some parents did not recognize hypoglycemia's symptoms or understand its treatment protocol. One safe way to treat hypoglycemia is by glucagon injection, but some parents injected the glucagon incorrectly, causing hyper- or hypoglycemia. For instance, Par-k-11 revealed that she had incorrectly injected her child with an entire dose of glucagon. Failure to resolve such mistakes in the administration of insulin can cause serious short- or long-term complications [20]

\section{Misapplication of correction factors.}

This category addresses how parents learn about the number of units of rapid-acting insulin that reduce BG levels, also known as the insulin sensitivity factor. Parents were asked about their understanding of how to calculate the insulin CF. In most cases, parents reported that the CF was calculated by professionals and not through their learning the calculation. For instance, Par-a-6 said: 
[Well, the calculation was already done by the doctor. They told me before figuring it. It was better that I get the calculation ready, since I did not know how to calculate it.] However this does not mean that all parents depended on the professionals. Some noticed that the estimated doses did not bring their children's BG down and made efforts to learn how to calculate the CF. Yet they lacked the confidence to do this.

[I used a book (to understand the calculation of the insulin correction factor). I read available information related to the calculation. But I don't know if it is right or not!] Par-k-14

The source of this problem is the professionals who decide on the extent of information parents require to treat their children's hyperglycemia. This investigation revealed that parents were not given all of the guidance necessary to appropriately treat their children's condition. In some cases, professionals intentionally prescribed a fixed number of units by following the Pediatric Insulin Sliding Scale and used that number for all cases which was not suitable for all circumstances.

Dia-Edu-K-8: [I taught them roughly based on every one unit needed to drop $100 \mathrm{mg} / \mathrm{dl}$.]

Researcher: [Did you teach each case differently based on their required units?]

Dia-Edu-K-8: [No! I generalized this number for all of them.]

Researcher: [Why?]

Dia-Edu-K-8: [Because it is difficult to teach each case separately].

A lack of clear guidelines for diabetes educators to follow when providing parents with appropriate treatment information is one of the key causes of parental errors [16]. A possible solution would be designing clear guidelines for professionals and parents to ensure better understanding, proper estimations of calculated insulin doses, and appropriate expected results.

Theme 2. Mistakes parents make with injection tools and techniques

This theme covers parents' detection of faults in insulin pen components and mistakes in injection techniques. It consists of two categories: firstly, parents' wasting insulin by testing faulty devices; and secondly, parents injecting insulin wrongly.

Parents' wasted time and insulin by testing faulty devices

This category addresses parents' wasting time to discover errors in insulin delivery. An analysis of the data showed that mistakes in recognizing the delivery of insulin occurred through technical faults. After giving correction doses, some parents noticed that their children's blood glucose did not change. They then checked the insulin pens used and realized that the dial was technically loose and the insulin was not being delivered correctly. In response to these technical errors, the parents tested the devices for the next correction. Par-k-10 stated:

[For the second correction, I gave my child insulin from the same insulin pen, but her BG was high. I changed the needle heads for her pens, and I dropped insulin, maybe four drops, to test the needles. So, I made a dose adjustment and noticed that the dial was broken] Par-K-10.

Checking the insulin flow with each new or used pen was a response to faulty pens, such as those with broken dose selection dials. Some parents stated that when their pens' buttons were pushed to release the insulin, the flow of insulin from the pens did not move. These parents threw the non-working devices away without extracting and reusing the insulin in their reservoirs. Parents are unfamiliar with how to easily recognize flaws in insulin delivery devices when they first occur (Ambler and Cameron) [14], and it is clear that teaching them the techniques for checking these flaws will help to ensure the correct delivery of the insulin they are given.

Mistakes with insulin injecting sites

This category addresses parents' mistakes with reference to injection sites. The data collected showed that many parents practiced the wrong insulin injection techniques. This predicament was commented on by one mother when asked about the injection sites.

[Many parents do not know where to inject and how to inject the needle and doing things badly] Par-k-22

An educator explained the reason why parents made mistakes when injecting insulin.

[We hope our education clinic is a perfect place. I mean it should be prepared with a new tools and modern equipment for injection techniques. We are three diabetes educators here and sharing the same room. I have a patient and my colleague has her at the same time. Dia-edu-k-9

Researcher: what are other problems?

[We don't have visible and educational things that can show it to the patient and parents. So, if we have a doll with complication of wrong injection techniques and another normal doll without complication. So, they will see the difference. Also, both educated and the illiterate parents will receive information in better way] Dia-edu-k-9

With the number of newly diagnosed cases increasing, one diabetes educator (Dia-edu-k-1) discovered that parents of previously diagnosed children had learned injection techniques incorrectly and needed to be re-educated. The unprepared clinics and the absence of ensuring good diabetes education procedures for previous parents, affected parents' understanding of insulin injection techniques and this caused mistakes. Thus parents need to learn new injection techniques that suit their current environment.

Parents' mistakes in self-administration errors led to another complication. For example, the local subcutaneous fat abnormalities, lipodystrophy and hypertrophy were major causes of hyperglycemia and examined by professionals. Most parents did not act on this issue immediately because they had been given no information since the early stages of diagnosis. This is illustrated by their continuing to inject their children in the same sites they did before the new information was discovered. Once lipoatrophy was examined, these parents had the option of following some procedures, e.g., rotating techniques. Few parents with more than seven years' experience with T1D corrected their mistakes. They searched the internet, asked other parents of T1D children for 
the appropriate injection techniques, and Barnea-Goraly et al [30] note that parents of newly and recently diagnosed T1D children learn about the rotating technique from their hospitals after diagnosis. However, this error commonly still occurs for most parents as one endocrinologist (Endo-k-4] pointed out.

These repeated errors involving injection sites and mistakes were common for both professionals and parents. A common injection challenge arose from needles puncturing tiny blood vessels. For instance, one mother reported bleeding after lifting an insulin injection for five seconds for correction. She reacted by questioning the professionals in-depth and predicting future mistakes, e.g. can she giving additional doses? The diabetes educator (Dia-Edu-K-4) received a number of questions from parents about bleeding issues caused by the needles used for corrections doses. Some parents had made many mistakes involving changes in injection sites and techniques.

Insulin leakage was a form of mistake at the injection site that many parents made. Immediate withdrawal (waiting 0 seconds) after injecting the needle into the skin frequently happened. Participants expressed apprehension about "wet spots" when giving their children injections. However, they recognized that this was insulin after smelling it. One parent (Par-K-16 M) described the scent as a "hospital smell." This was an indicator that insulin had leaked out of the skin. This parent corrected her mistake by questioning the diabetes educator during last her visits (e.g. for how long should she leave the injection in the skin. However, such parents had not learned a set of procedures in the earlier stages of diagnosis so that they could understand such issues.

\section{Discussion}

\subsection{Diabetes Education and Treatment of Hyperglycemia in Children}

Fleisher and Ludwig [2] assert that T1D occurs when the pancreas is no longer able to produce adequate insulin after the destruction of beta cells. The T1D is common to young children under the age of ten years and a small percentage of young adults around twenty years. The ability to control the condition among children is challenging and parents endure devastating circumstances. Truong [11] contends that diabetic management in children requires a proper understanding of the condition and a good management plan. Apart from medical therapy, controlling hyperglycemia in children requires other management techniques such as engagement in physical activities and a balanced diet. Hanas [7] states that the growing prevalence of diabetes in adults and children initiates a growing demand for its prevention. However, people are naïve about this chronic condition and must be provided with the necessary knowledge, information, skills, and strategies to mitigate it.

Having access to diabetes education is important to patients parents and caregivers. Diabetes educators inform patients and their caregivers about the different types of diabetes and how they are managed [36]. They help diabetic and their caregivers improve clinical practice in order to live a better quality of life (QoL). The hyperglycemia caused by large amounts of glucose in the blood can be managed through continual access to self-management education, awareness and support to prevent future complications and risks of long-term complications. In the past, diabetes was managed by medication engaging in physical exercises, and consuming a balanced diet. However, techniques of managing diabetes in the contemporary have become complex. Although the techniques apply the same, many issues have changed. For example, diabetes diet management has shifted from the physician to the individual controlling the meal plan. According to Magaji and Johnston [10], patients have a greater role in controlling the condition rather than the medical team.

Brook and Dattani [16] state that fathers and mothers are responsible for every day management of T1D children. They should have an sufficient understanding, skills, information and strategies for managing the condition of their children. Parents need to be educated on monitoring blood glucose, pressure, and how to measure glycated hemoglobin [42]. Despite the process having a important affect on family life, mothers and fathers have to ensure diabetic children are well protected. Effective and good quality diabetes management needs a knowledgeable caregiver and having a strong medical team to help patients make critical decisions [23]. Diabetic adolescents should also be engaged in the process of managing diabetes, and this requires rigorous effort from the patient. Having knowledge alone is not enough to manage the condition but the diabetics should individually engage in active self-care. According to Streisand et al [43], fathers and mothers have a duty to create the diabetes management plan in order to help their child manage the condition.

\subsection{Giving Insulin Injections}

Giving insulin injections to patients is one of the biggest challenges experienced by parents [44]. Insulin is administered through insulin pens, injectors, syringes, and pumps. The parents should seek advice from the medical team on the appropriate technique. Adolescents always require parental support in order to meet the insulin dosage regimen [45]. This demonstrates that fathers and mothers have the responsibility to ensure their child is injected with the correct amount of insulin on a daily basis. Syringe and insulin pens are commonly used because they are accessible for every patients. They hold a variant amount of insulin depending on the size. Fathers and mothers should aware of the dosage and seek advice and information from the diabetic team when necessary [46]. Furthermore, parents should be involved when available in order to build mutual support so that the diabetic child is protected.

Parents should acquire information how to rotate children's injection sites when dosages are administered on a daily basis [31]. Continuous inject of the same site leads to lipodystrophy in which fat builds up under the skin causing lumps that interfere with insulin absorption. The injections can be rotated 
in many parts of the body like as abdomen, thighs,, and arms. The injection sites must be one inch apart to avoid physical injuries to patients and also ease absorption [47]. Insulin is more absorbed in the abdomen and is the recommended site of injection. The top and outer parts of the thigh are good injection sites while on the arm, the fatty area between the shoulder and the elbow are also appropriate. Children and parents should adhere to insulin injection steps when administering dosages [48]. Measuring the dosage should be done correctly and the injection should be administered gently or one to two inches of skin. To conclude, authors recommend the adoption of 'Diabetes Awareness' and made possible by diabetes education.

The child needs to depend on the suggestions from the diabetes health care team. Approaches to treatment and insulin dosages given will differ depending on the level of diabetes and the learner. Dungan, et al [36] write that treatment of diabetic youth involves ensuring the child participates in physical activities, eating a balanced diet, taking insulin as prescribed and checking blood sugar level regularly. Parents have to ensure their children inject insulin dosages as prescribed in order to maintain their BS level in a healthy range [11].

\section{Future Use of the Work}

This project is significant because it presents critical information on diabetes management in children specially T1D. The content in this study is helpful to endocrinologists, diabetes educators, health department and companies' representatives. The research is also a good source of diabetes education more specially awareness to parents and patients. The aim of this research and data collected revealed that most fathers and mothers do not know how to manage the condition in children at home. Majority of parents have to rely on the diabetes team to properly administer insulin dosage to children [44]. The issues discovered in this study on T1D management in children and adolescents will enable diabetic educators and health department and companies' representatives to establish good policies in addressing the issue in society. Parents handling diabetic children will also receive relevant information on managing the condition before it becomes worse.

According to Hulkower, et al [9], health ministries in many countries have the responsibility to create awareness to people on diabetes management. The study has revealed that many parents have a poor understanding of insulin injection technique The technique is somehow complicated to fathers and mothers who have never used it before on their children. This study advises American, Australian and Saudi Arabian ministries or departments of health to ensure that proper policies are established to address the challenges pointed out here. Endocrinologists will have the chance to understand more about T1D in children and improve the ways of restoring the normal balance of hormones in the body. Geller and Delbello [49] contend that diabetes is an endocrine system disorder and endocrinologists always need information for further research.

\section{Conclusion}

Interviews with parents of T1D children, and ancillary medical staff have shown that there are two main mistakes that parents make and these are also important themes: 1: Misestimating and misunderstanding insulin correction doses; and 2: Using injection tools and techniques. The first theme has two aspects: 1- Underestimated and overdosed insulin units and misused Glucagon injection, and 2- Misapplication of correction factors. The second theme consists of two aspects: 1- Parents wasted time and insulin by testing faulty devices, and 2- Mistakes with insulin injecting sites. It is clear from the data that parents' misunderstanding of insulin dosage leads to several mistakes that demonstrate that most of them do not know how to handle the child. The study has revealed that most parents are scared of injecting their child and therefore need a medical professional who will highlight errors in insulin dosage and increase parents' confidence. The process can be daunting and very technical, and consequently parents find it hard to inject their own child.

Initial education has been shown to be ineffective in the long-term and this indicates the need for follow up sessions. More effort needs to be put into reducing fear and increasing confidence in parents. This can be done by assuming that parents will need follow up education in which they are supervised in calculating and administering correct dose sizes and in the correct sites. A change in the medical system could be a first step in solving the issue. For example, professionals need to help parents differentiate the types of insulin, how they work and how they are administered to diabetic children. In addition, professionals need to help parents to be confident, trust the devices and show them how to check if devices are faulty. The ability to check faulty devices could be done during the provided diabetes educational visits.

There are several techniques used to regulate the blood sugar level of diabetic children. Administering insulin is critical and the medical system needs to encourage parents to adhere to a set routine with insulin dosage. Accordingly, professionals need to simplify for the parent or caregiver the process of assessing the blood sugar level of the child.

The data reveals a need to ensure that parents have the skills to deal with the child, and ensure that he or she engages in physical exercise and that they consume a balanced diet.

The mistakes made by parents can lead to serious emotional, psychological and financial consequences that make some parents give up. When parents are overwhelmed by the outcomes, the need to consult the health team for therapy is critical. Finally, parents need to be updated on the current issues on diabetes management in children, and provided with continual learning on how to better care for their T1D child.

\section{Acknowledgements}

The authors thank the volunteering participants including: (parents, endocrinologists, diabetes educators, and companies' representatives); and governmental and private diabetic institutions such as, firstly, the Saudi Arabian Ministry of 
Health $(\mathrm{MOH})$ represented by Dr. Tareef Al Aama and Dr. Mohammed Yahia Alharbi, and secondly, Diabetes Victoria.

\section{Conflict of Interest}

All the authors do not have any possible conflicts of interest.

\section{References}

[1] Jayakumar, R., Bhavani, N., \& Pavithran, P. (2013). Diabetes in children and adolescents. New Delhi: JP Medical Ltd, Diabetes in Children and Adolescents. 2013: JP Medical Ltd.

[2] Fleisher, G., \& Ludwig, S. (2010). Textbook of pediatric emergency medicine. Philadelphia: Lippincott Williams \& Wilkins, Textbook of pediatric emergency medicine. 2010: Lippincott Williams \& Wilkins.

[3] Daneman, D., Type 1 diabetes. The Lancet, 2006. 367 (9513): p. $847-858$.

[4] Roemer, J. A. d. a. g. t. r. a. c. w. d. V. A. D. A., Roemer, J. (2011). America] $\mathrm{n}$ diabetes association guide to raising a child with diabetes. Virginia: American Diabetes Assoiation.

[5] Wolfsdorf, J., N. Glaser, and M. A. Sperling, Diabetic Ketoacidosis in Infants, Children, and Adolescents A consensus statement from the American Diabetes Association. Diabetes care, 2006. 29 (5): p. 1150-1159.

[6] Kanikarla-Marie, Preeti, and Sushil K. Jain, Hyperketonemia and ketosis increase the risk of complications in type 1 diabetes. Free Radical Biology and Medicine, 2016. 95: p. 268-277.

[7] Hanas, R. T. d. i. c., adolescents, and young adults: How to become an expert on your own diabetes. Bristol: Class Publishing Ltd, Type 1 diabetes in children, adolescents, and young adults: how to become an expert on your own diabetes. 2007: Class Publishing Ltd.

[8] Vehik, K., Hamman, R., Lezotte, D., Norris, J., Klingensmith, G., $\&$ Bloch, C. (2007). Increasing incidence of type 1 diabetes in 0 to 17 year old Colorado youth. Diabetes Care, 30 (3), 503-509, Increasing incidence of type 1 diabetes in 0 -to 17 -year-old Colorado youth. Diabetes care, 2007. 30 (3): p. 503-509.

[9] Hulkower, R., Pollack, R., \& Zonszein, J. (2014). Understanding hypoglycemia in hospitalized patients. Diabetes Management, 4 (2), 165-176, Understanding hypoglycemia in hospitalized patients. Diabetes management (London, England), 2014. 4 (2): p. 165.

[10] Magaji, V. and J. M. Johnston, Inpatient management of hyperglycemia and diabetes. Clinical Diabetes, 2011. 29 (1): p. 3-9.

[11] Truong, T. H., et al., Errors in the Administration Technique of Insulin Pen Devices: A Result of Insufficient Education. Diabetes Therapy, 2017.8 (2): p. 221-226.

[12] Trief, P. M., et al., Incorrect insulin administration: a problem that warrants attention. Clinical Diabetes, 2016. 34 (1): p. 25-33.

[13] Umpierrez, G. E., et al., Management of hyperglycemia in hospitalized patients in non-critical care setting: an endocrine society clinical practice guideline. The Journal of Clinical
Endocrinology \& Metabolism, 2012.97 (1): p. 16-38.

[14] Ambler, G., \& Cameron, F. (2010). Caring for diabetes in children and adolescents. Wales: Combined Children's Diabetes Services Caring for diabetes in children and adolescents. 2010.

[15] reiser, J. G. c. a. n. J. o. P. a. E. N., 35 (12), 671-672, Glycemic control and nutrition. 2011, Sage Publications Sage CA: Los Angeles, CA.

[16] Brook, C., \& Dattani, M. (2012). Handbook of clinical pediatric endocrinology. New Jersey: John Wiley\& Sons, Handbook of clinical pediatric endocrinology. 2012: John Wiley \& Sons.

[17] Pettitt, D., Talton, J., Dabelea, D., Divers, J., Imperatore, G., \& Lawrence J. (2009). The search for diabetes in youth study. Diabetes Care, 37 (2), 402-408. doi: 10.2337/dc13-1838, The search for diabetes in youth study. Diabetes Care, 2009. 37 (2), p. $402-408$.

[18] Niedel, A., Traynor, M., Mckee, M., \& Grey, M. (2012). Parallel vigilance: Parents' dual focus following diagnosis of type 1 diabetes mellitus in their young child. Health, $0(0), 1-20$, Parallel vigilance: parents' dual focus following diagnosis of Type 1 diabetes mellitus in their young child. Health:, 2012. 17 (3): p. 246-265.

[19] Young-Hyman, D., \& Peyrot, M. (2012). Psychosocial care for people with diabetes. Virginia: American Diabetes Association, Psychosocial care for people with diabetes. 2012: American Diabetes Association.

[20] Turchin, A., Matheny, M., \& Shubina, M. (2009). Hypoglycemia and clinical outcomes in patients with diabetes hospitalized in the general ward. Diabetes Care, 32 (7), 1153-1157, Hypoglycemia and clinical outcomes in patients with diabetes hospitalized in the general ward. Diabetes Care, 2009. 32 (7): p. 1153-1157.

[21] Draznin, B. M. d. a. h. i. t. h. s. A. c. s. g. V. A. D. A., Managing Diabetes and Hyperglycemia in the Hospital Setting: A Clinician's Guide. 2016: American Diabetes Association.

[22] Cryer, P. H., functional brain failure, and brain death. Journal of Clinical Investigation, 117 (4), 868-870, Hypoglycemia, functional brain failure, and brain death. The Journal of clinical investigation, 2007. 117 (4): p. 868-870.

[23] Patton, S., Dolan, L., Smith, L., Brown, M., \& Powers, S. (2013). Examining mealtime behaviors in families of young children with type 1 diabetes on intensive insulin therapy. Eating Behaviour, 14 (4), 464-467, Examining mealtime behaviors in families of young children with type 1 diabetes on intensive insulin therapy. Eating behaviors, 2013. 14 (4): p. 464-467.

[24] Taylor, R., Williams, S., Farmer, V., \& Taylor, B. (2013). Changes in physical activity over time in young children: a longitudinal study using accelerometers. PLoS One, 8 (11), 81567 , Changes in physical activity over time in young children: a longitudinal study using accelerometers. PLoS One, 2013. 8 (11): p. e81567.

[25] Schatz, D., Haller, M., \& Atkinson, M. (2010). Type 1 diabetes, an issue of endocrinology and metabolism clinics of North America, E-book. Amsterdam: Elsevier Health Sciences, Type 1 Diabetes, An Issue of Endocrinology and Metabolism Clinics of North America, E-Book. Vol. 39. 2010: Elsevier Health Sciences. 
[26] Faulkner, M., Family Responses to Children with Diabetes and Their Influence on Self-Care., Family responses to children with diabetes and their influence on self-care. Journal of Pediatric Nursing, 1996. 11 (2): p. 82-93

[27] Bruno, A., Gregori, D., \& Caropreso, A. (2008). Normal glucose values are associated with a lower risk of mortality in hospitalized patients. Diabetes Care, 31 (11), 2209-2210, Normal glucose values are associated with a lower risk of mortality in hospitalized patients. Diabetes Care, 2008. 31 (11): p. $2209-2210$

[28] Moghissi, E., Korytkowski, M., \& Dinardo, M. (2009). American association of clinical endocrinologists and american diabetes consensus statement on inpatient glycemic conrol. Diabetes Care. 32 (6), 1119-1131, American Association of Clinical Endocrinologists and American Diabetes Association consensus statement on inpatient glycemic control. Diabetes care, 2009. 32 (6): p. 1119-1131.

[29] Sullivan-Bolyai, S. L., M. (2011). Parent mentor perspectives on providing social support to empower parents. The Diabetes Educator, 37, (3), 34-43, Parent mentor perspectives on providing social support to empower parents. The Diabetes Educator, 2011. 37 (1): p. 35-43.

[30] Barnea-Goraly, N., Raman, M., Mazaika, P., Marzelli, M., Hershley, T., \& Weinzimer, S. (2014). Alterations in white matter structure in young children with type 1 diabetes. Diabetes Care, 37 (2), 332-340. doi:10.2337/dc13-1388, Alterations in white matter structure in young children with type 1 diabetes. Diabetes Care, 2014. 37 (2): p. 332-340.

[31] Mertig, R. N. s. g. t. t. d. s. m., second edition. New York: Spring Publishing Company, Nurses' Guide to Teaching Diabetes Self-management. 2011: Springer Publishing Company.

[32] Gagnum, V., Stene, L. C., Leivestad, T., Joner, G. and Skrivarhaug, T., 2017. Long-term mortality and end-stage renal disease in a type 1 diabetes population diagnosed at age 15-29 years in Norway. Diabetes care, 40 (1), pp. 38-45., Long-term mortality and end-stage renal disease in a type 1 diabetes population diagnosed at age 15-29 years in Norway. Diabetes care, 2017. 40 (1): p. 38-45.

[33] Dabelea, D., Stafford, J. M., Mayer-Davis, E. J., D’Agostino, R., Dolan, L., Imperatore, G., Linder, B., Lawrence, J. M., Marcovina, S. M., Mottl, A. K. and Black, M. H., 2017. Association of type 1 diabetes vs type 2 diabetes diagnosed during childhood and adolescence with complications during teenage years and young adulthood. Jama, 317 (8), pp. 825-835.

[34] Bruttomeso, D., \& Grassi, D. (2014). Technological advances in the treatment of type 1 diabetes. Basel: Karger Medical and Scientific Publishers, Technological advances in the treatment of type 1 diabetes. 2014: Karger Medical and Scientific Publishers.

[35] Korytkowski, M., Salata, R.,\& Koerbel, G. (2009). Insulin therapy and glycemic control in hospitalized patients with diabetes during ennteral nutrition therapy: A randomized controlled clinical trial. Diabetes Care, 32 (3), 594-596, Insulin therapy and glycemic control in hospitalized patients with diabetes during enteral nutrition therapy: a randomized controlled clinical trial. Diabetes care, 2009. 32 (4): p. 594-596.

[36] Dungan, K., Braithwaite, S., \& Preiser, J. (2009). Stress hyperglycemia. Lancet, 373 (9677), 1798-1807, Stress hyperglycaemia. The Lancet, 2009. 373 (9677): p. 1798-1807.

[37] Steele, C., Hagopian, W., \& Gitelman, S. (2004). Insulin secretion in type 1 diabetes. Diabetes, 53 (4), 426-433, Insulin secretion in type 1 diabetes. Diabetes, 2004. 53 (2): p. 426-433.

[38] Bienso, R., Ringholm, S., \& Kiilerich, K. (2012). GLUT4 and glycogen synthase are key players in bed rest-induced insulin resistance. Diabetes. 61 (10), 1090-1099, GLUT4 and Glycogen Synthase Are Key Players in Bed Rest-Induced Insulin Resistance. Diabetes, 2012. 61 (5): p. 1090-1099.

[39] Marzelli, M., Masaika, P., Barnea-Goraly, N., Hershey, T., Tsalikian, E.,\& Tamborlane, W. (2014). Neuroanatomical correlates of dysglycemia in young children with type 1 diabetes. Diabetes, 63 (1), 343-353, Neuroanatomical correlates of dysglycemia in young children with type 1 diabetes. Diabetes, 2014. 63 (1): p. 343-353.

[40] Trochim, W. M. and J. P. Donnelly, 'Research methods knowledge base', 2001.

[41] Browne, G. J. and M. B. Rogich, An empirical investigation of user requirements elicitation: Comparing the effectiveness of prompting techniques. Journal of Management Information Systems, 2001. 17 (4): p. 223-249.

[42] Gasmavov, A., \&Umipierrez, G. (2012). Medical nutrition therapy in hospitalized patients with diabetes. Current Diabetes Reports. 12 (3), 92-100, Medical nutrition therapy in hospitalized patients with diabetes. Current diabetes reports, 2012. 12 (1): p. 93-100.

[43] Streisand, R., Mackey, E., Elliot, B., Mednick, L., Slaughter, I., \& Turek, J. (2008). Parental anxiety and depression associated with caring for a child newly diagnosed with type 1 diabetes: Opportunities for education and counseling. Patient Education Cou, Parental anxiety and depression associated with caring for a child newly diagnosed with type 1 diabetes: opportunities for education and counseling. Patient education and counseling, 2008. 73 (2): p. 333-338.

[44] Seshiah, V. M. c. o. d. A. E. H. S., Microvascular Complications of Diabetes-ECAB. 2009: Elsevier Health Sciences.

[45] Jiang, H., Stryer, D., Friedman, B., \& Andrews, R. (2003). Multiple hospitalizations for patients with diabetes. Diabetes Care, 26 (5), 1421-1426, Multiple hospitalizations for patients with diabetes. Diabetes care, 2003. 26 (5): p. 1421-1426.

[46] Hilliard, M., Monaghan, M., Cogen, F., \& Streisand, R. (2011). Parent stress and child behavior among young children with type 1 diabetes. Child Care Health Developmemt, 37 (2), 224-232 Parent stress and child behaviour among young children with type 1 diabetes. Child: care, health and development, 2011. 37 (2): p. 224-232.

[47] Herrin, M. N. c. i. t. t. o. e. d. L. R., Nutrition counseling in the treatment of eating disorders. 2012: Routledge.

[48] Trief, P. M., Cibula, D., Rondriguez, E., Akel, B. \& Weinstok, R. S. (2016). Incorrect insulin administration: a problem that warrants attention. Clinical Diabetes, 34, 25-33., Incorrect insulin administration: a problem that warrants attention. Clinical Diabetes, 2016. 34 (1): p. 25-33.

[49] Geller, B., \& Delbello, M. (2008). Treatment of bipolar disorder in children and adolescents. New York: Guilford Press, Treatment of bipolar disorder in children and adolescents. 2008: Guilford Press. 